\title{
Risk of COVID-19 for Patients with Cancer: A Narrative Overview
}

\author{
Mohamed Amine Baba ${ }^{1,2^{*}}(\mathbb{D})$, Youssef Bouchriti ${ }^{1,3}(\mathbb{D})$, Abderrahmane Achbani ${ }^{1,4}(\mathbb{D})$, Ahmed Kharbach ${ }^{1,5}$ (D), \\ Hasnaa Sine ${ }^{6}$ (D), Aziz Naciri ${ }^{7}$ \\ ${ }^{1}$ High Institute of Nursing Professions and Technical Health, Agadir, Morocco \\ ${ }^{2}$ National School of Applied Sciences, Ibn Zohr University, Agadir, Morocco \\ ${ }^{3}$ Faculty of sciences Ibn Zohr, Agadir, Morocco \\ ${ }^{4}$ Laboratory of Cell Biology and Molecular Genetics Department of Biology, Faculté of Sciences, Ibn Zohr University, Agadir, Morocco \\ ${ }^{5}$ Laboratory of Biostatistics Clinical Research and Epidemiology (LBRCE) Faculty of Medicine and Pharmacy of Rabat, MOHAMED V \\ University of Rabat, Morocco \\ ${ }^{6}$ Medical Biology, Human and experimental pathology and Environment, Faculty of Medicine and Pharmacy of Rabat, MOHAMED V \\ University of Rabat, Morocco \\ ${ }^{7}$ High Institute of Nursing Professions and Technical Health, Laayoun, Morocco \\ * Corresponding author: Mohamed Amine Baba E-mail: babamedamine2@gmail.com ORCID: 0000-0002-6660-9527 \\ Received: 30 April 2020 Accepted: 5 May 2020
}

\begin{abstract}
In March 2020, the WHO declared COVID-19 as a pandemic. At present, the curative vaccine has not yet been developed, older age and higher comorbidity levels are associated with more severe COVID-19 symptoms. Indeed, the cancer community currently faces many difficult questions.
\end{abstract}

Keywords: Coronavirus, COVID-19, cancer, 2019-nCoV, pneumonia, oncology

\section{INTRODUCTION}

The emergence of coronavirus disease 2019 (COVID-19) has caused a global public health emergency [1]. This new type of respiratory illness is characterized by rapid human-tohuman transmission, having achieved pandemic spread [2]. COVID-19 is associated with presentations ranging from asymptomatic infections to severe viral pneumonia, acute respiratory distress syndrome and death [3].

There are currently no therapeutics or vaccines available and, presumably, no pre-existing immunity in the population. Genetic analysis of the full-length genome sequences revealed SARS-CoV- 2 to be most closely related to a known bat coronavirus termed BatCoV RaTG13, suggesting bats as the likely origin [4].

Cancer patients represent a population more exposed to the risk of infection and possible complications, since immunocompromised hosts differ from the general population for several reasons [5]. First, they harbor pathogens for a longer period of time, which makes them more infectious. second, it is difficult to maintain them in quarantine because of their frequent medical needs. Finally, the course of the infection does not generally follow the natural history of the disease as observed in apparently immunocompetent hosts. However, a study has shown the 
case fatality rate reached $5.6 \%$ among cancer patients compared with $2.3 \%$ in the general population [3].

Also knowing that some cancer patients may have diagnostic results similar to those of SAR-CoV2 infection and may be misleading [6] then clinical information in cancer patients with COVID-19 is limited and many questions asked about the clinical characteristics and the management of this population.

\section{CLINICAL CHARACTERISTICS OF COVID-19-INFECTED CANCER PATIENTS}

A cohort of cancer patients with COVID-19 have clinical characteristics similar to those in the general population, with the exception of anemia and hypoproteinemia, which were frequently found in this cohort [7]. Anemia and hypoproteinemia can be considered a major consequence of nutritional deterioration in cancer patients, which can affect immunocompetence and increase sensitivity to respiratory pathogens.

Patients with cancer had a higher risk of developing severe events (intensive care unit admission, invasive ventilation, or death) compared with patients without cancer, several studies have shown that patients who had received last antitumour treatment within 14 days had a statistically significant increased risk of developing severe events including chemotherapy, radiotherapy, targeted therapy, and immunotherapy, combined with chemotherapy . [7, 8] The symptom of dyspnea has appeared much earlier since the start of COVID-19 infection in patients with lung cancer compared to the general population [9], which requires special measures for this population and in hospitals specializing in oncology.

For good management of cancer patients during this pandemic, it is recommended that treatment strategies likely to cause immunosuppression should be avoided for stable patients or else reduce the doses.

In this perspective, a recent study has proposed a conceptual framework for prioritizing the use of radiotherapy and systemic treatments during the COVID-19 pandemic, in order to minimize harm, postponing cancer treatments might be associated with some risk, although these risks will need to be considered in light of the magnitude of potential benefits, the impact of waiting times on outcomes and competing patient-level and system-level priorities.[10] However, the most difficult therapeutic decision concerns metastatic diseases, for these patient's treatment delays may lead to worsening performance status and loss of the window to treat [11]. some authors recommend, that patients cancer patients remain in the observation room and isolated from other patients at least 7 days before treatment for cancerous tumors [7]. It also adds that individual protection must be reinforced, with a massive screening strategy through early diagnostic tests in cancer patients because of the similarity of symptoms between the infection and the underlying disease, in particular in lung cancer patients and patients with lung metastases, as the diagnosis of COVID-19 may be delayed in this situation.

In conclusion, the safety and management of cancer patients with severe acute respiratory syndrome 2 (SARSCoV-2) is urgent and oncology establishments must establish an emergency plan to adapt to the situation without interrupt their activity.

\section{DECLARATION OF CONFLICT OF INTEREST}

The authors received no financial support for the research and/or authorship of this article. There is no conflict of interest.

\section{REFERENCES}

1. Sohrabi $C$, et al. World Health Organization declares global emergency: A review of the 2019 novel coronavirus (COVID-19). International Journal of Surgery 2020. (doi: 10.1016/j.jjsu.2020.02.034).

2. Yu J, et al. SARS-CoV-2 transmission in cancer patients of a tertiary hospital in Wuhan. medRxiv. 2020. (doi: 10.1101/2020.02.22.20025320).

3. Wu Z, McGoogan JM.M. Characteristics of and important lessons from the coronavirus disease 2019 (COVID-19) outbreak in China: summary of a report of 72314 cases from the Chinese Center for Disease Control and Prevention. Jama. 2020; 323(13): 1239-42. (doi: 10.1001/jama.2020.2648).

4. Zhou $P$, et al. A pneumonia outbreak associated with a new coronavirus of probable bat origin. Nature, 2020; 579(7798): 270-3. (doi: 10.1038/s41586-020-2012-7).

5. Schreiber RD, Old LJ, Smyth M.J. Cancer immunoediting: integrating immunity's roles in cancer suppression and promotion. Science, 2011; 331(6024): 1565-70. (doi: 10.1126/science.1203486).

6. Zhu W, et al. The differential diagnosis of pulmonary infiltrates in cancer patients during the outbreak of the 2019 novel coronavirus disease. Chinese Journal of Oncology, 2020; 42: E008-E008. (doi: 10.3760/cma.j.cn112152-20200303-00166). 
7. Zhang $L$, et al. Clinical characteristics of COVID-19infected cancer patients: A retrospective case study in three hospitals within Wuhan, China. Annals of Oncology, 2020. (doi: 10.1016/j.annonc.2020.03.296).

8. Liang W, et al. Cancer patients in SARS-CoV-2 infection: a nationwide analysis in China. The Lancet Oncology, 2020: 21(3): 335-7. (doi: 10.1016/S1470-2045(20)30096-6).

9. Yan X, et al. Clinical management of lung Cancer patients during the outbreak of 2019 novel coronavirus disease (COVID-19). Zhongguo Fei Ai Za Zhi, 2020; 23(3). (doi: 10.3779/j.issn.1009-3419.2020.03.02).
10. Hanna TP, Evans GA, Booth CM. Cancer, COVID-19 and the precautionary principle: prioritizing treatment during a global pandemic. Nature Reviews Clinical Oncology, 2020; 17(5): 268-70. (doi: 10.1038/s41571-0200362-6).

11. Ueda $M$, et al. Managing cancer care during the COVID19 pandemic: agility and collaboration toward a common goal. Journal of the National Comprehensive Cancer Network, 2020; 1(aop): 1-4. (doi: 10.6004/jnccn.2020.7560). 